\title{
An analogue of the Bernstein-Walsh lemma in Jordan regions of the complex plane
}

Fahreddin G Abdullayev* and Naciye Pelin Özkartepe

${ }^{*}$ Correspondence:

fabdul@mersin.edu.tr;

fahreddinabdullayev@gmail.com Faculty of Arts and Science,

Department of Mathematics, Mersin

University, Mersin, 33343, Turkey

\begin{abstract}
In this paper we continue to study two-dimensional analogues of Bernstein-Walsh estimates for arbitrary Jordan domains.
\end{abstract}

MSC: Primary 30A10; 30C10; secondary 41A17

Keywords: algebraic polynomials; conformal mapping; Bernstein lemma

\section{Introduction and main results}

Let $G \subset \mathbb{C}$ be a finite region, with $0 \in G$, bounded by a Jordan curve $L:=\partial G, \Delta:=\{w$ : $|w|>1\}, \Omega:=\operatorname{ext} \bar{G}$ (with respect to $\overline{\mathbb{C}}$ ). Let $w=\Phi(z)$ be the univalent conformal mapping of $\Omega$ onto the $\Delta$ normalized by $\Phi(\infty)=\infty, \Phi^{\prime}(\infty)>0$, and $\Psi:=\Phi^{-1}$.

Let $\wp_{n}$ denote the class of arbitrary algebraic polynomials $P_{n}(z)$ of degree at most $n \in \mathbb{N}$. Let $A_{p}(G), p>0$, denote the class of functions $f$ which are analytic in $G$ and satisfy the condition

$$
\|f\|_{A_{p}(G)}:=\left(\iint_{G}|f(z)|^{p} d \sigma_{z}\right)^{1 / p}<\infty
$$

where $\sigma$ denotes a two-dimensional Lebesgue measure.

When $L$ is rectifiable, let $\mathcal{L}_{p}(L), p>0$, denote the class of functions $f$ which are integrable on $L$ and satisfy the condition

$$
\|f\|_{\mathcal{L}_{p}(L)}:=\left(\int_{L}|f(z)|^{p}|d z|\right)^{1 / p}<\infty .
$$

From the well-known Bernstein-Walsh lemma [1, p.101], we see that

$$
\left|P_{n}(z)\right| \leq|\Phi(z)|^{n}\left\|P_{n}\right\|_{C(\bar{G})}, \quad z \in \Omega
$$

For $R>1$, let us set $L_{R}:=\{z:|\Phi(z)|=R\}, G_{R}:=\operatorname{int} L_{R}, \Omega_{R}:=\operatorname{ext} L_{R}$. Then (1.1) can be written as follows:

$$
\left\|P_{n}\right\|_{C\left(\bar{G}_{R}\right)} \leq R^{n}\left\|P_{n}\right\|_{C(\bar{G})}
$$

Hence, setting $R=1+\frac{1}{n}$, according to (1.2), we see that the $C$-norm of a polynomial $P_{n}(z)$ in $\bar{G}_{R}$ and $\bar{G}$ is equivalent, i.e., the norm $\left\|P_{n}\right\|_{C\left(\bar{G}_{R}\right)}$ increases with no more than a constant with respect to $\left\|P_{n}\right\|_{C(\bar{G})}$.

\section{Springer}

○2013 Abdullayev and Özkartepe; licensee Springer. This is an Open Access article distributed under the terms of the Creative Commons Attribution License (http://creativecommons.org/licenses/by/2.0), which permits unrestricted use, distribution, and reproduction in any medium, provided the original work is properly cited. 
In the case when $L$ is rectifiable, a similar estimate of (1.2) type in space $\mathcal{L}_{p}(L)$ was obtained in [2] as follows:

$$
\left\|P_{n}\right\|_{\mathcal{L}_{p}\left(L_{R}\right)} \leq R^{n+\frac{1}{p}}\left\|P_{n}\right\|_{\mathcal{L}_{p}(L)}, \quad p>0
$$

The Berstein-Walsh type estimation for regions with quasiconformal boundary [3, p.97] in the space $A_{p}(G), p>0$, is contained in [4]:

$$
\left\|P_{n}\right\|_{A_{p}\left(G_{R}\right)} \leq c_{2} R^{*^{n+\frac{1}{p}}}\left\|P_{n}\right\|_{A_{p}(G)}, \quad p>0
$$

where $R^{*}:=1+c_{1}(R-1)$ and $c_{1}>0, c_{2}=c_{2}\left(c_{1}, p, G\right)>0$ are constants. Therefore, if we choose $R=1+\frac{c_{3}}{n}$, then (1.4) we can see that the $A_{p}$-norm of polynomials $P_{n}(z)$ in $G_{R}$ and $G$ is equivalent.

In this work, we study a problem similar to (1.4) in $A_{p}(G), p>0$, for regions with arbitrary Jordan boundary.

Now we can state our new result.

Theorem 1.1 Let $p>0 ; G$ be a Jordan region. Then, for any $P_{n} \in \wp_{n}, R_{1}=1+\frac{1}{n}$ and arbi$\operatorname{trary} R, R>R_{1}$, we have

$$
\left\|P_{n}\right\|_{A_{p}\left(G_{R}\right)} \leq c_{4} R^{n+\frac{2}{p}}\left\|P_{n}\right\|_{A_{p}\left(G_{R_{1}}\right)},
$$

where $c_{4}=\left(\frac{2}{e^{p}-1}\right)^{\frac{1}{p}}\left[1+O\left(\frac{1}{n}\right)\right], n \rightarrow \infty$.

The sharpness of (1.5) can be seen from the following remark:

Remark 1.1 For any $n=1,2, \ldots$, there exist a polynomial $P_{n}^{*} \in \wp_{n}$, region $G^{*} \subset \mathbb{C}$ and number $R>R_{1}=1+\frac{1}{n}$ such that

$$
\left\|P_{n}^{*}\right\|_{A_{p}\left(G_{R}^{*}\right)} \geq\left(\frac{2}{e^{p}-1}\right)^{\frac{1}{p}} R^{n+\frac{2}{p}}\left\|P_{n}^{*}\right\|_{A_{p}\left(G_{R_{1}}^{*}\right)} .
$$

\section{Some auxiliary results}

Let $G \subset \mathbb{C}$ be a finite region bounded by the Jordan curve $L$. Let $L_{R}:=\{z:|\Phi(z)|=R, R>1\}$, $G_{t}:=\operatorname{int} L_{t}, \Omega_{t}:=\operatorname{ext} L_{t}$.

We note that, throughout this paper, $c_{1}, c_{2}, \ldots$ (in general, different in different relations) are positive constants.

Lemma 2.1 Let $p>0 ; f$ be an analytic function in $|z|>1$ and have a pole of degree at most $n, n \geq 1$ at $z=\infty$. Then, for any $R_{1}$ and $R>R_{1}$, we have

$$
\|f\|_{A_{p}\left(R_{1}<|z|<R\right)} \leq\left(\frac{R^{n p+2}-R_{1}^{n p+2}}{R_{1}^{n p+2}-1}\right)^{\frac{1}{p}}\|f\|_{A_{p}\left(1<|z|<R_{1}\right)} .
$$

Proof The function $g(z):=\frac{f(z)}{z^{n}}$ is analytic in $|z|>1$ and continuous in $|z| \geq 1$. Applying Hardy's convexity theorem [5, p.9: Th.1.5], for any arbitrary $R_{1}$ and $R\left(R>R_{1}\right)$, and $\rho, s$ 
such that $R_{1} \leq \rho<R, 1<s \leq R_{1}$, we can write

$$
\begin{aligned}
& \int_{|z|=\rho}\left|\frac{f(z)}{z^{n+\frac{1}{p}}}\right|^{p}|d z| \leq \int_{|z|=R_{1}}\left|\frac{f(z)}{z^{n+\frac{1}{p}}}\right|^{p}|d z|, \\
& \int_{|z|=R_{1}}\left|\frac{f(z)}{z^{n+\frac{1}{p}}}\right|^{p}|d z| \leq \int_{|z|=s}\left|\frac{f(z)}{z^{n+\frac{1}{p}}}\right|^{p}|d z|,
\end{aligned}
$$

respectively. Thus,

$$
\begin{aligned}
& \int_{|z|=\rho}|f(z)|^{p}|d z| \leq \rho^{n p+1} \int_{|z|=R_{1}}\left|\frac{f(z)}{z^{n+\frac{1}{p}}}\right|^{p}|d z|, \\
& s^{n p+1} \int_{|z|=R_{1}}\left|\frac{f(z)}{z^{n+\frac{1}{p}}}\right|^{p}|d z| \leq \int_{|z|=s}|f(z)|^{p}|d z| .
\end{aligned}
$$

Integrating (2.4) over $\rho$ from $R_{1}$ to $R$, and (2.5) over $s$ from 1 to $R_{1}$, we get

$$
\begin{aligned}
& \int_{R_{1}}^{R} \int_{|z|=\rho}|f(z)|^{p}|d z| d \rho \leq \frac{1}{n p+2}\left(R^{n p+2}-R_{1}^{n p+2}\right) \int_{|z|=R_{1}}\left|\frac{f(z)}{z^{n+\frac{1}{p}}}\right|^{p}|d z|, \\
& \frac{1}{n p+2}\left(R_{1}^{n p+2}-1\right) \int_{|z|=R_{1}}\left|\frac{f(z)}{z^{n+\frac{1}{p}}}\right|^{p}|d z| \leq \int_{1}^{R_{1}} \int_{|z|=s}|f(z)|^{p}|d z| d s .
\end{aligned}
$$

After calculation we have

$$
\iint_{R_{1}<|z|<R}|f(z)|^{p} d \sigma_{z} \leq \frac{R^{n p+2}-R_{1}^{n p+2}}{R_{1}^{n p+2}-1} \iint_{1<|z|<R_{1}}|f(z)|^{p} d \sigma_{z}
$$

and we see that (2.1) is true.

Corollary 2.2 Under the assumptions of Lemma 2.1 for $R_{1}=1+\frac{1}{n}$, we have

$$
\|f\|_{A_{p}\left(R_{1}<|z|<R\right)} \leq c_{1} R^{n+\frac{2}{p}}\|f\|_{A_{p}\left(1<|z|<R_{1}\right)}
$$

where $c_{1}:=c_{1}(p, n)=\left(\frac{1}{e^{p}-1}\right)^{\frac{1}{p}}\left[1+O\left(\frac{1}{n}\right)\right], n \rightarrow \infty$.

Proof Let us put

$$
S^{p}:=S^{p}\left(R, R_{1}, n, p\right):=\frac{R^{n p+2}-R_{1}^{n p+2}}{R_{1}^{n p+2}-1}=R^{n p+2} \cdot \frac{1-\left(\frac{R_{1}}{R}\right)^{n p+2}}{R_{1}^{n p+2}-1},
$$

and taking $R_{1}=1+\frac{1}{n}$, we have

$$
S^{p}=R^{n p+2} \cdot \frac{1-\left(\frac{R_{1}}{R}\right)^{n p+2}}{\left(1+\frac{1}{n}\right)^{n p+2}-1} \leq \frac{R^{n p+2}}{\left(1+\frac{1}{n}\right)^{n p+2}-1} .
$$

According to the right-hand side of the well-known estimation (see, for example, [6, p.52 (Problem 170)])

$$
\frac{e}{2 n+2}<e-\left(1+\frac{1}{n}\right)^{n}<\frac{e}{2 n+1}, \quad n=1,2, \ldots
$$


Abdullayev and Özkartepe Journal of Inequalities and Applications 2013, 2013:570

Page 4 of 7

http://www.journalofinequalitiesandapplications.com/content/2013/1/570

we have

$$
\left(1+\frac{1}{n}\right)^{n p+2} \geq\left(1+\frac{1}{n}\right)^{n p} \geq\left(e-\frac{e}{2 n+1}\right)^{p}=e^{p} \cdot\left(1-\frac{1}{2 n+1}\right)^{p} \geq\left(\varepsilon_{n} \cdot e\right)^{p},
$$

where

$$
\frac{2}{3} \leq \varepsilon_{n}:=1-\frac{1}{2 n+1} \rightarrow 1, \quad n \rightarrow \infty .
$$

Therefore

$$
S^{p} \leq \frac{1}{\left(\varepsilon_{n} e\right)^{p}-1} R^{n p+2}=R^{n p+2} \frac{1}{e^{p}-1}\left[1+O\left(\frac{1}{n}\right)\right], \quad n \rightarrow \infty .
$$

From (2.8) and (2.11) we complete the proof.

Remark 2.1 For the polynomial $Q_{n}(z)=z^{n}, R_{1}=1+\frac{1}{n}$ and any $R>R_{1}$,

$$
\left\|Q_{n}\right\|_{A_{p}\left(R_{1}<|z|<R\right)} \geq c_{2} R^{n+\frac{2}{p}}\left\|Q_{n}\right\|_{A_{p}\left(1<|z|<R_{1}\right)},
$$

where $c_{2}:=c_{2}(p, n):=\left(\frac{1}{e^{p}-1}\right)^{\frac{1}{p}}\left[1-O\left(\frac{1}{n}\right)\right], n \rightarrow \infty$.

Proof Really, from (2.6) we get

$$
S^{p}=R^{n p+2} \cdot \frac{1-\left(\frac{R_{1}}{R}\right)^{n p+2}}{\left(1+\frac{1}{n}\right)^{n p+2}-1}=R^{n p+2} \cdot \frac{1-\delta_{n}}{\left(1+\frac{1}{n}\right)^{n p+2}-1},
$$

where

$$
\delta_{n}:=\left(\frac{R_{1}}{R}\right)^{n p+2} \rightarrow 0, \quad n \rightarrow \infty
$$

According to the left-hand side of (2.9), we obtain

$$
\begin{aligned}
\left(1+\frac{1}{n}\right)^{n p+2} & =\left(1+\frac{1}{n}\right)^{n p}\left(1+\frac{1}{n}\right)^{2} \leq\left(e-\frac{e}{2 n+2}\right)^{p} \eta_{n} \\
& =e^{p} \cdot\left(1-\frac{1}{2 n+2}\right)^{p} \eta_{n} \leq e^{p} \cdot \eta_{n},
\end{aligned}
$$

where

$$
\eta_{n}:=\left(1+\frac{1}{n}\right)^{2} \rightarrow 1, \quad n \rightarrow \infty
$$

Therefore,

$$
\begin{aligned}
S^{p} & \geq R^{n p+2} \cdot \frac{1-\delta_{n}}{\eta_{n} e^{p}-1} \\
& =R^{n p+2} \cdot\left[\frac{1}{\eta_{n} e^{p}-1}-\frac{\delta_{n}}{\eta_{n} e^{p}-1}\right]
\end{aligned}
$$




$$
\begin{aligned}
& =R^{n p+2} \cdot\left\{\frac{1}{e^{p}-1}\left[1-O\left(\frac{1}{n}\right)\right]-O\left(\delta_{n}\right)\right\} \\
& =R^{n p+2} \cdot \frac{1}{e^{p}-1}\left[1-O\left(\frac{1}{n}\right)\right], \quad n \rightarrow \infty .
\end{aligned}
$$

Corollary 2.3 For $f \equiv P_{n}$, we have

$$
\left\|P_{n}\right\|_{A_{p}(|z|<R)} \leq c_{3} R^{n+\frac{2}{p}}\left\|P_{n}\right\|_{A_{p}\left(|z|<R_{1}\right)}
$$

where $c_{3}:=c_{3}(p, n):=\left(\frac{2}{e^{p}-1}\right)^{\frac{1}{p}}\left[1+O\left(\frac{1}{n}\right)\right], n \rightarrow \infty$.

Proof Really, (2.1) implies, for any $f \equiv P_{n}$,

$$
\left\|P_{n}\right\|_{A_{p}\left(R_{1}<|z|<R\right)}^{p} \leq S^{p} \cdot\left\|P_{n}\right\|_{A_{p}\left(1<|z|<R_{1}\right)}^{p}
$$

Adding $\left\|P_{n}\right\|_{A_{p}\left(|z|<R_{1}\right)}^{p}$ to the both sides, we obtain

$$
\begin{aligned}
\left\|P_{n}\right\|_{A_{p}(|z|<R)}^{p} & \leq S^{p} \cdot\left\|P_{n}\right\|_{A_{p}\left(1<|z|<R_{1}\right)}^{p}+\left\|P_{n}\right\|_{A_{p}\left(|z|<R_{1}\right)}^{p} \\
& \leq 2 \max \left\{S^{p}, 1\right\} \cdot\left\|P_{n}\right\|_{A_{p}\left(|z|<R_{1}\right)}^{p} .
\end{aligned}
$$

Passing to the limit as $R_{1}=1+\frac{1}{n} \rightarrow 1$, from (2.11) we obtain

$$
\left\|P_{n}\right\|_{A_{p}(|z|<R)}^{p} \leq \frac{2}{e^{p}-1}\left[1+O\left(\frac{1}{n}\right)\right] \cdot R^{n p+2}\left\|P_{n}\right\|_{A_{p}\left(|z|<R_{1}\right)}^{p} .
$$

\section{Proof of the theorem}

Proof First of all, let us convince ourselves that for the proof of (1.5) it is sufficient to show the fulfilment of estimation

$$
\left\|P_{n}\right\|_{A_{p}\left(G_{R} \backslash G_{R_{1}}\right)} \leq c R^{n+\frac{2}{p}}\left\|P_{n}\right\|_{A_{p}\left(G_{R_{1}} \backslash G\right)}
$$

for some constant $c=c\left(p, R_{1}\right)>0$ independent of $R$ and $n$. Really, let (3.1) be true. Then

$$
\left\|P_{n}\right\|_{A_{p}\left(G_{R} \backslash G_{R_{1}}\right)}^{p} \leq c^{p} R^{n p+2}\left\|P_{n}\right\|_{A_{p}\left(G_{R_{1}} \backslash G\right)}^{p} .
$$

Now, we will add to both sides $\left\|P_{n}\right\|_{A_{p}\left(G_{R_{1}}\right)}^{p}$ :

$$
\begin{aligned}
\left\|P_{n}\right\|_{A_{p}\left(G_{R}\right)}^{p} & \leq c^{p} R^{n p+2}\left\|P_{n}\right\|_{A_{p}\left(G_{R_{1}} \backslash G\right)}^{p}+\left\|P_{n}\right\|_{A_{p}\left(G_{R_{1}}\right)}^{p} \\
& \leq c^{p} R^{n p+2}\left\|P_{n}\right\|_{A_{p}\left(G_{\left.R_{1} \backslash G\right)}\right.}^{p}+c^{p} R^{n p+2}\left\|P_{n}\right\|_{A_{p}\left(G_{R_{1}}\right)}^{p} \\
& =2 c^{p} R^{n p+2}\left\|P_{n}\right\|_{A_{p}\left(G_{R_{1}}\right)}^{p} .
\end{aligned}
$$

Therefore,

$$
\left\|P_{n}\right\|_{A_{p}\left(G_{R}\right)} \leq 2^{\frac{1}{p}} c R^{n+\frac{2}{p}}\left\|P_{n}\right\|_{A_{p}\left(G_{R_{1}}\right)} .
$$


Now, let us make a proof of (3.1).

For the $p>0$, let us set

$$
f_{n}(w):=P_{n}(\Psi(w))\left[\Psi^{\prime}(w)\right]^{\frac{2}{p}}, \quad w=\Phi(z) .
$$

The function $f_{n}$ is analytic in $\Delta$ and has a pole of degree at most $n$ at $w=\infty$. Then, according to Lemma 2.1, we have

$$
\left\|f_{n}\right\|_{A_{p}\left(R_{1}<|w|<R\right)}^{p} \leq S\left(R, R_{1}, n, p\right)\left\|f_{n}\right\|_{A_{p}\left(1<|w|<R_{1}\right)}^{p},
$$

where

$$
S^{p}:=\frac{R^{n p+2}-R_{1}^{n p+2}}{R_{1}^{n p+2}-1}=R^{n p+2} \cdot \frac{1-\left(\frac{R_{1}}{R}\right)^{n p+2}}{R_{1}^{n p+2}-1} .
$$

Then

$$
\begin{aligned}
\iint_{G_{R} \backslash G_{R_{1}}}\left|P_{n}(z)\right|^{p} d \sigma_{z} & =\iint_{R_{1}<|w|<R}\left|f_{n}(w)\right|^{p} d \sigma_{w} \\
& \leq S^{p} \iint_{1<|w|<R_{1}}\left|f_{n}(w)\right|^{p} d \sigma_{w} \\
& \leq R^{n p+2} \cdot \frac{1}{R_{1}^{n p+2}-1} \iint_{G_{R_{1} \backslash G}\left|P_{n}(z)\right|^{p} d \sigma_{z} .}
\end{aligned}
$$

Therefore,

$$
\iint_{G_{R}}\left|P_{n}(z)\right|^{p} d \sigma_{z} \leq 2 R^{n p+2} \cdot \frac{1}{R_{1}^{n p+2}-1} \iint_{G_{R_{1}}}\left|P_{n}(z)\right|^{p} d \sigma_{z} .
$$

Taking $R_{1}=1+\frac{1}{n}$, from (2.9) and (2.11) we get

$$
\frac{1}{R_{1}^{n p+2}-1}=\frac{1}{e^{p}-1}\left[1+O\left(\frac{1}{n}\right)\right], \quad n \rightarrow \infty
$$

Now, from (3.4) and (3.5) we complete the proof.

\subsection{Proof of the remark}

Proof Let $P_{n}^{*}=z^{n}, G^{*}=B:=\{z:|z|<1\}$ and $R \leq \frac{8 e^{p}}{e^{p}-1}$. Then

$$
\begin{aligned}
\left\|P_{n}^{*}\right\|_{A_{p}\left(G_{R}^{*}\right)}^{p} & =\iint_{|z|<R}\left|z^{n}\right|^{p} d \sigma_{z} \\
& =R^{n p+2} \cdot R_{1}^{-(n p+2)}\left\|P_{n}^{*}\right\|_{A_{p}\left(G_{R_{1}}^{*}\right)}^{p} \\
& =\frac{R}{R_{1}^{2} \cdot R_{1}^{n p}} \cdot R^{n p+2}\left\|P_{n}^{*}\right\|_{A_{p}\left(G_{R_{1}}^{*}\right)}^{p} .
\end{aligned}
$$


For $R_{1}=1+\frac{1}{n}$, from (2.9) we obtain

$$
\begin{aligned}
& \left(1+\frac{1}{n}\right)^{n p} \leq\left(e-\frac{e}{2 n+2}\right)^{p} \leq e^{p} \\
& \left(1+\frac{1}{n}\right)^{2} \leq 4
\end{aligned}
$$

Then

$$
\frac{R}{R_{1}^{n p+2}} \geq \frac{R}{4 e^{p}}
$$

and

$$
\left\|P_{n}^{*}\right\|_{A_{p}\left(G_{R}^{*}\right)}^{p} \geq \frac{R}{4 e^{p}} \cdot R^{n p+2}\left\|P_{n}^{*}\right\|_{A_{p}\left(G_{R_{1}}^{*}\right)}^{p} .
$$

In particular, for $R=\frac{8 e^{p}}{e^{p}-1}$ we have

$$
\left\|P_{n}^{*}\right\|_{A_{p}\left(G_{R}^{*}\right)}^{p} \geq \frac{2}{e^{p}-1} \cdot R^{n p+2}\left\|P_{n}^{*}\right\|_{A_{p}\left(G_{R_{1}}^{*}\right)}^{p} .
$$

Competing interests

The authors declare that they have no competing interests.

\section{Authors' contributions}

All authors contributed equally and significantly in writing this paper. All authors read and approved the final manuscript.

Received: 17 May 2013 Accepted: 7 November 2013 Published: 02 Dec 2013

\section{References}

1. Walsh, JL: Interpolation and Approximation by Rational Functions in the Complex Domain. Am. Math. Soc., Providence (1960)

2. Hille, E, Szegö, G, Tamarkin, JD: On some generalization of a theorem of A Markoff. Duke Math. J. 3, $729-739$ (1937)

3. Lehto, O, Virtanen, Kl: Quasiconformal Mapping in the Plane. Springer, Berlin (1973)

4. Abdullayev, FG: On the some properties of the orthogonal polynomials over the region of the complex plane (Part III). Ukr. Math. J. 53(12), 1934-1948 (2001)

5. Duren, PL: Theory of $H^{P}$ Spaces. Academic Press, San Diego (1970)

6. Polya, G, Szegö, G: Problems and Theorems in Analysis I. Nauka, Moscow (1978) (Russian edition)

10.1186/1029-242X-2013-570

Cite this article as: Abdullayev and Özkartepe: An analogue of the Bernstein-Walsh lemma in Jordan regions of the complex plane. Journal of Inequalities and Applications 2013, 2013:570

\section{Submit your manuscript to a SpringerOpen ${ }^{\ominus}$ journal and benefit from:}

- Convenient online submission

Rigorous peer review

- Immediate publication on acceptance

- Open access: articles freely available online

- High visibility within the field

- Retaining the copyright to your article 